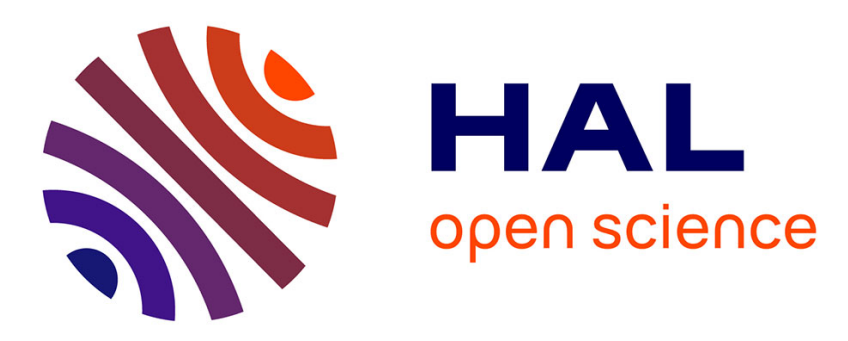

\title{
Post-processing of Solar Irradiance Forecasts from WRF Model at Reunion Island
}

\author{
Hadja Maïmouna Diagne, Mathieu David, John Boland, Nicolas Schmutz, \\ Philippe Lauret
}

\section{- To cite this version:}

Hadja Maïmouna Diagne, Mathieu David, John Boland, Nicolas Schmutz, Philippe Lauret. Postprocessing of Solar Irradiance Forecasts from WRF Model at Reunion Island. Energy Procedia, 2014, 57, pp.1364 - 1373. 10.1016/j.egypro.2014.10.127 . hal-01089749

\section{HAL Id: hal-01089749 \\ https://hal.science/hal-01089749}

Submitted on 2 Dec 2014

HAL is a multi-disciplinary open access archive for the deposit and dissemination of scientific research documents, whether they are published or not. The documents may come from teaching and research institutions in France or abroad, or from public or private research centers.
L'archive ouverte pluridisciplinaire HAL, est destinée au dépôt et à la diffusion de documents scientifiques de niveau recherche, publiés ou non, émanant des établissements d'enseignement et de recherche français ou étrangers, des laboratoires publics ou privés. 


\title{
Post-processing of solar irradiance forecasts from WRF Model at Reunion Island
}

\author{
Maïmouna Diagne ${ }^{\mathrm{a}, \mathrm{b}, *}$, Mathieu David ${ }^{\mathrm{b}}$, John Boland ${ }^{\mathrm{c}}$, Nicolas Schmutz ${ }^{\mathrm{a}}$, \\ Philippe Lauret ${ }^{\mathrm{b}}$ \\ ${ }^{a}$ REUNIWATT company, Saint-Denis, Réunion, France \\ ${ }^{b}$ PIMENT Laboratory, University of La Réunion, 15 Avenue Cassin, 97715 Saint-Denis, Réunion, France \\ ${ }^{c}$ Centre for Industrial and Applied Mathematics and the Barbara Hardy Institute, University of South Australia
}

\begin{abstract}
An efficient use of solar energy production requires reliable forecast information on surface solar irradiance. This article aims at providing a model output statistics (MOS) method of improving solar irradiance forecasts from Weather Research and Forecasting (WRF) Model.

The WRF model was used to produce one year of day ahead solar irradiance forecasts covering Reunion Island with an horizontal resolution of $3 \mathrm{~km}$. These forecasts are refined with a Kalman filter using high quality ground measurements. Determination of the relevant data inputs for the Kalman filter method is realized with a bias error analysis. Solar zenith angle and the clear sky index, among others, are used for this analysis.

Accuracy of the method is evaluated with a comprehensive testing procedure using different error metrics. Kalman filtering appears to be a viable method in order to improve the solar irradiance forecasting.
\end{abstract}

(C) 2014 Published by Elsevier Ltd. This is an open access article under the CC BY-NC-ND license

(http://creativecommons.org/licenses/by-nc-nd/3.0/).

Selection and/or peer-review under responsibility of ISES.

Keywords: Solar irradiance, Post processing, Kalman filter, WRF, Clear Sky Index, Solar Zenith Angle

\section{Introduction}

At Reunion Island, the threshold of $30 \%$ of intermittent renewables energy (wind power and photovoltaic) in the energetic mix has been achieved. Incoming solar radiation is the most important meteorological factor that influences the power production of a solar energy power plant. There is an

\footnotetext{
* Corresponding author.E-mail address: maimouna.diagne@reuniwatt.com.
} 
increasing need for accurate solar forecasting methods at different forecast horizons, say hourly or daily. It is helpful for operational control and optimization of some energy systems.

Solar power production is highly variable due to the reflection of solar radiation on clouds. Because of the intermittent character of the resource, it is important to know how much solar energy is produced in the next days for the entire management of total power production from different sources and power delivery to customers. Thus the importance of a forecast of solar power production is evident for the entire energy industry.

Two approaches allow the forecasting of solar irradiance. The first one consists of statistical models. It is based on historical data. The second approach consists of numerical weather prediction (NWP) models. NWP models can be global (ECMWF, GFS ...) or local (WRF, NAM ...) [1]. Post processing methods are frequently applied to refine the output of NWP models like model output statistics (MOS) in Lorenz [2].

The use of NWP to estimate GHI has been extensively tested [3], [4], [5]; however, differences over a specific location usually arise in regions with changeable weather and typical partially cloudy days [5].

In this work, hourly global horizontal irradiance (GHI) is forecasted with WRF model [6] for day ahead. Next Kalman filtering, a post processing method, is applied to improve GHI forecasts hour by hour. Our objective is to increase the accuracy of the solar irradiance forecast at Reunion island for use in an operational setting.

\section{Methods}

The solar irradiance forecast method follows several steps as illustrated by Figure 1. First, we download forecasts of the Global Forecast System (GFS) model from the NCEP web site [7]. Next GFS data are used as input to the WRF model in order to downscale the spatial and temporal resolution of GFS forecasts. GHI forecasts from WRF are compared to ground measurements in order to evaluate forecast accuracy. In the last step, we apply a post processing method like Kalman filter in order to improve WRF forecasts.

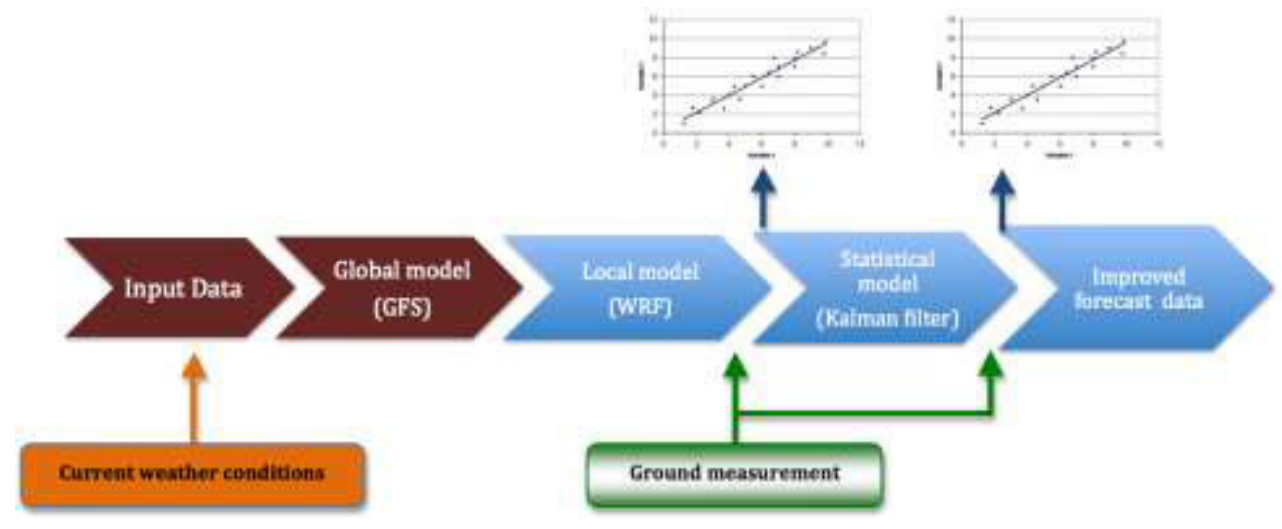

Figure 1: Improvement of WRF forecasts through a Kalman filter post-processing technique

\subsection{WRF forecasts}

A regional NWP model called Weather Research and Forecast (WRF) model is used to forecast hourly surface solar irradiance on a day ahead basis for one year from January 2011 to December 2011. The WRF configuration includes three two-way nested domains with horizontal resolutions of 27, 9 and $3 \mathrm{~km}$ 
(Figure 2) in order to obtain a high resolution forecast. A variable distribution of one hundred vertical levels with more levels near the surface, was applied. WRF generates forecasts of 30 hours ahead. During our study, we systematically delete the first 6 hours because we consider that the WRF model needs time to stabilize. Horizon forecast between 0 and $24 \mathrm{~h}$ of the next day is used. The temporal resolution is 6 minute. The spatial resolution reaches about $3 \mathrm{~km}$ at the grid center, at Saint Pierre in Reunion Island. Forecasts were extracted for the variables and grid points of interest using the NCL programming language [8].
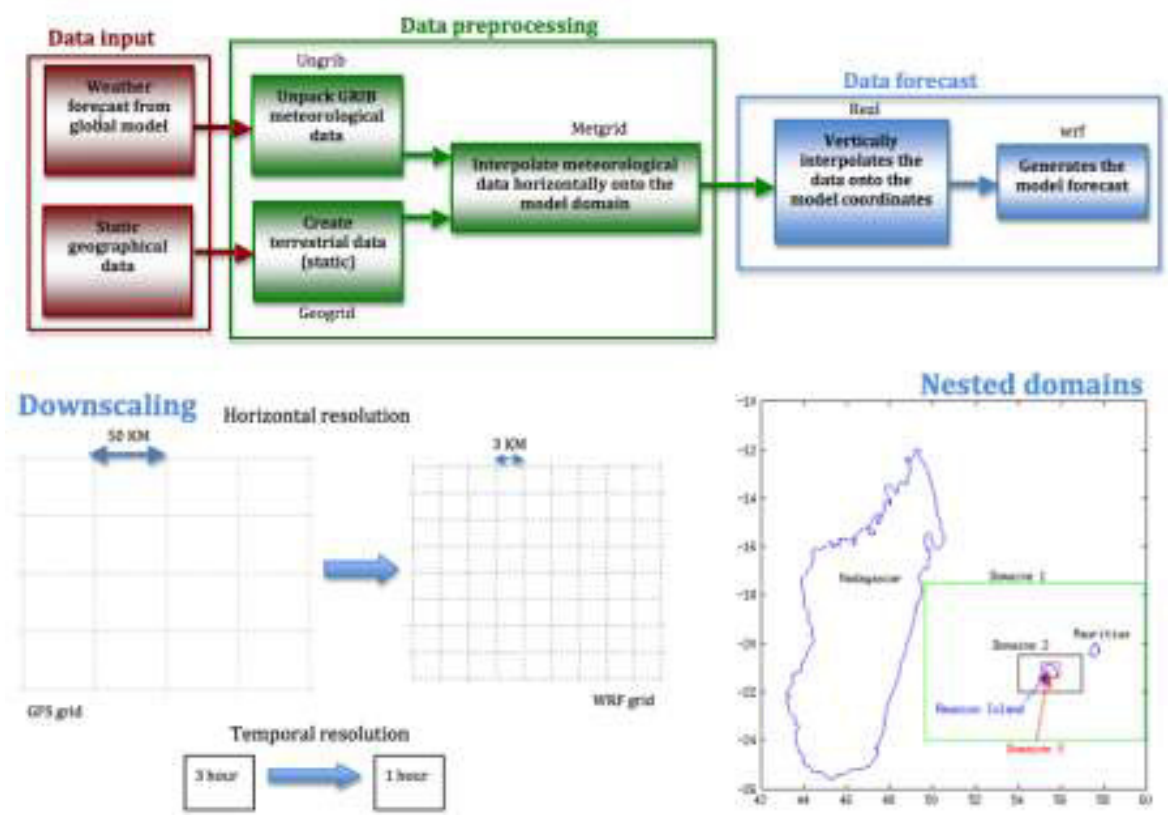

Figure 2: Downscaling of temporal and spatial resolution of GFS data with WRF model

Initial and boundary conditions were obtained from GFS forecasts. GFS data are available up to four times a day, at $0 \mathrm{UTC}, 6 \mathrm{UTC}, 12 \mathrm{UTC}$, and $18 \mathrm{UTC}$, and at time steps of $3 \mathrm{~h}$ and horizontal resolution of $0.5^{\circ} \times 0.5^{\circ}$ [7]. Only forecasts originating at $06 \mathrm{UTC}$ in Grib2 format were considered here because these are the most relevant for day-ahead PV forecasting in Reunion Island. Currently, our objective is to produce forecasts by $17 \mathrm{~h} 00$ for each hour of the following day, which means that 06 UTC forecasts are the most recent forecasts available prior to the $17 \mathrm{~h} 00$ deadline. The day-ahead forecast period corresponds to forecast horizons of 21 to $45 \mathrm{~h}$ ahead in the GFS 06 UTC forecast because Reunion Island has a $4 \mathrm{~h}$ jet lag. Elevation and land cover data were provided by the digital terrain model from the United States Geological Survey [9].

In spite of this high resolution forecast, during cloudy days some discrepancies between model results and measurements were observed. These differences are mainly because of the difficulty to forecast the development of clouds and transport over a single location. 


\subsection{Ground data measurements}

Modeled GHI results were compared against ground measurements. The ground station is located at Saint Pierre $\left(21^{\circ} 20 \mathrm{~S} ; 55^{\circ} 29 \mathrm{E}\right)$ at the south of Reunion Island.

The station measures direct, sky diffuse, and GHI each six second among others, reported as 1-min averages.

\subsection{Forecast accuracy}

The accuracy measures used to evaluate solar forecasts vary. Benchmarking of solar forecasts has been examined in David et al. [10]. Following [10], criteria used to evaluate the forecast accuracy are the mean bias error (MBE), the root mean squared error (RMSE) and the mean absolute error (MAE) defined as:

$R M S E=\sqrt{\frac{1}{n} \cdot \sum_{i=1}^{n}\left(x_{p r e d, i}-x_{o b s, i}\right)^{2}}$

$M B E=\frac{1}{n} \cdot \sum_{i=1}^{n}\left(x_{p r e d, i}-x_{o b s, i}\right)$

$\left.M A E=\frac{1}{n} \cdot \sum_{i=1}^{n}\left|\left(x_{o b s, i}-x_{\text {pred }, i}\right)\right|\right)$

Where $x_{p r e d, i}$ and $x_{o b s, i}$ represent the ith valid forecast and observation pair, respectively. As the above definitions indicate, RMSE gives more weight to large errors, whereas MAE reveals the average magnitude of the error and MBE indicates whether there is a significant tendency to systematically overforecast or under-forecast. When comparing different models in the training year, RMSE was used as the metric for minimization, that is, forecasts were trained with the goal of reducing the largest errors.

Relative values of these metrics (rRMSE, rMAE and rMBE) are obtained by normalization to the mean ground measured irradiance of the considered period.

Persistence is used as a benchmark to our proposed models. A persistence forecast is simply the assumption that the value for the next time step is the same as the present value, i.e.

$$
\hat{X}_{t+1}=X_{t}
$$

To improve forecast accuracy, we apply several post-processing methods. Six minute WRF forecasts and one minute ground station data were respectively averaged hourly. GHI values were excluded from the analysis when these were outside a physically plausible range. Solar forecast evaluation was restricted to solar zenith angle ranging from 0 degrees to 80 degrees which corresponds to daylight period.

\subsection{Bias removal using a Kalman filter}

Kalman filtering is a two-step predictor-corrector method that corrects/updates the estimated forecast error using the last observed error. It has been used extensively in a number of areas, including post- 
processing of NWP model outputs. Recently, Pelland et al. [11] and Galanis et al. [12] applied Kalman filtering to bias removal in solar irradiance forecasts and wind speed forecasts respectively. Following these authors, we investigated bias removal for irradiance forecasts by exploring different variations of their approaches. The most satisfactory approach was found to be one where the bias depends linearly on the forecasted irradiance ( ) and the solar zenith angle (SZA). These variables are selected after the evaluation of the accuracy of several variations in the model parameters. Tests including several combinations of meteorological data like GHI, SZA, hour of the day, temperature, humidity, pressure, ... show better results with $\left(\mathrm{GHI}_{\text {forecast } . t}\right)$ and the solar zenith angle (SZA). These variables are selected after the evaluation of the accuracy of several variations in the model parameters. Tests including several combinations of meteorological data like GHI, SZA, hour of the day, temperature, humidity, pressure ... show better results with $\mathrm{GHI}_{\text {forecast.t }}$ and SZA. SZA data are obtained from Bird model [13].

Let $x_{\text {vred. } i}$ be the state of the forecast error at time step $\mathrm{t}$ that is to be predicted. The subscript pred, $\mathrm{t}$ is used to denote predictions for time $t$ based on information available up to time $(t-1)$. The system equation defines the time dependent evolution of $x_{t-1}$ by a persistence of the current bias.

$x_{\text {pred }, t}=x_{t-1}+w_{t}$

Here $w_{t}$ represents the random change from $t-1$ to $t$ and is assumed to be normally distributed with mean zero and variance $W_{t}$. The observable bias at time step t, $y_{t}$ is assumed to be noisy, with a normally distributed random error term of $v_{t}$ of variance $V_{t}$ :

$y_{t}=H_{t} x_{t}+v_{t}$

Here $H_{t}$ is a 3 column matrix. $H_{t}=\left[1, G H I_{\text {forecast } t} / 1000, S Z A\right]$

The objective is to get the best estimate of, $x_{t}$ which is termed $x_{\text {vred.t }}$, by projecting the error covariance ahead $P_{\text {vred t.t }}$.

$$
P_{\text {pred, } t}=P_{t-1}+W_{t-1}
$$

For updating the estimate $x_{t}$, when the observed bias $y_{t}$ becomes available, equation 8 is used:

$$
x_{t}=x_{\text {pred }, t}+K_{t}\left(y_{t}-H_{t} x_{\text {pred }, t}\right)
$$

Where $K_{t}$ is the Kalman gain and is determined by equation 9:

$$
K_{t}=P_{\text {pred }, t} H_{t}^{T}\left(H_{t} P_{p r e d, t} H_{t}^{T}+V_{t}\right)^{-1}
$$

And finally for updating the error covariance term equation 10 is used:

$$
P_{t}=\left(I_{2 \times 2}-K_{t} H_{t}\right) P_{p r e d, t}
$$


At any time $t$, the forecast bias can be estimated as:

bias $_{\text {pred }, t}=1000 . y_{\text {pred }, t}=1000 . H_{t} x_{\text {pred }, t}$

For a more complete description of Kalman filtering and its application in post processing see Pelland et al. [11].

The full set of variables used in the Kalman filter procedure is shown in Figure 3, as well as the equations used in the iterative predict and update algorithm and the initial values selected.

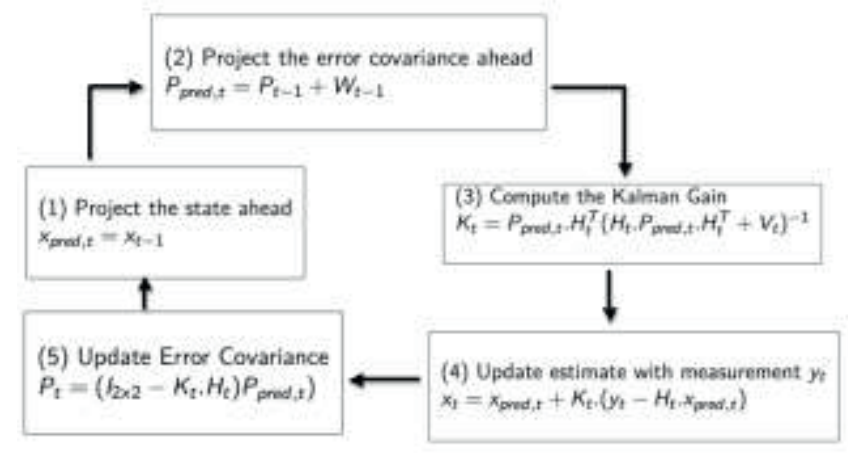

Figure 3: Kalman filtering algorithm (Extracted from Pelland et al. [11])

The initial values shown in Figure 3, were selected on the basis of tests over the 1-year training period (January 2011 to December 2011) for the 24-h-ahead horizon for the Saint Pierre forecasts, which showed significant bias. They were chosen to yield substantial bias reduction while also reducing RMSE. Similarly, the number $\mathrm{M}$ of training days over which $\mathrm{W}$ and $\mathrm{V}$ were calculated was selected by looking at the trade-off between bias removal and RMSE reduction over the 1-year training period.

\section{Results}

\subsection{WRF forecast evaluation}

The scatterplot of forecasted GHI vs measured GHI in Figure 4 shows that GHI forecast from WRF is not accurate.

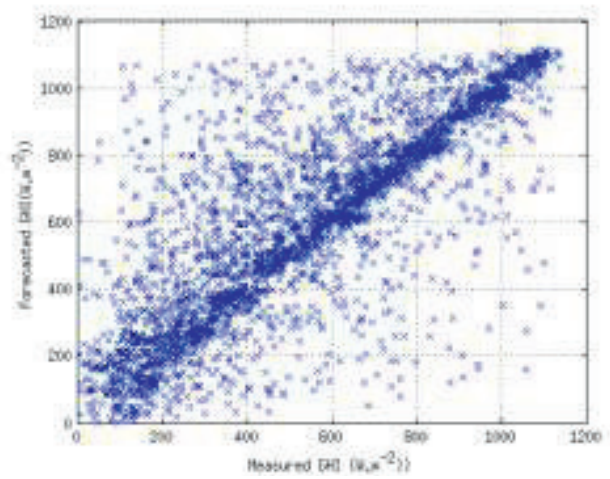

Figure 4: WRF GHI forecasted versus GHI measured 
The graphical representation of the MBE depending on the forecasted CSI and the SZA (Figure 5) allowed us to conclude that when a clear sky is forecasted then the model overestimates GHI. However if a cloudy sky is forecasted then the model underestimates. WRF forecast seems to be reliable for clear days and inaccurate for cloudy days. Accuracy evaluation gives a rRMSE of $35.2 \%$ and a rMBE of $9.5 \%$.

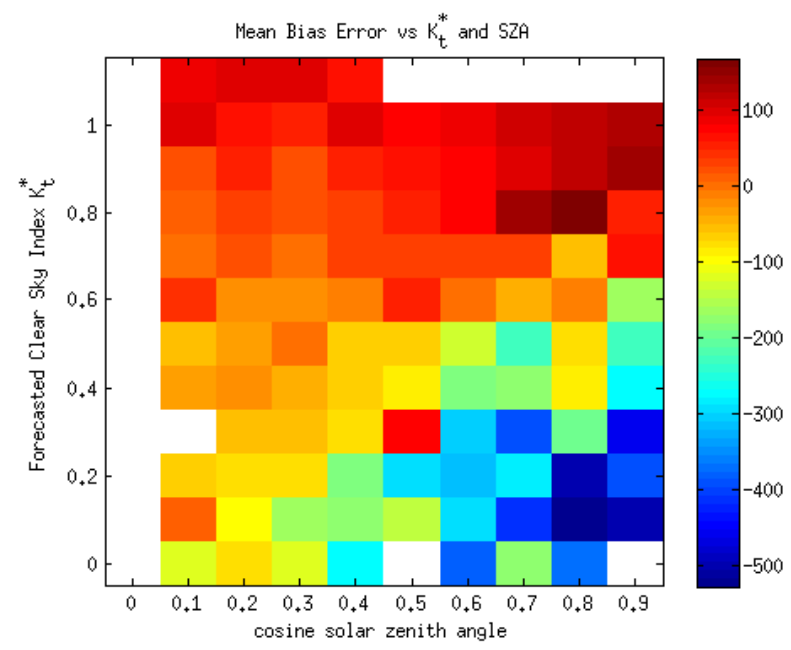

Figure 5: Variation of the bias with CSI forecasted and SZA

Accuracy evaluation depending on the hour of the day, Figure 6, show that the MBE is low during the morning. It corresponds to the period at Saint Pierre where there is high occurence of clear sky.

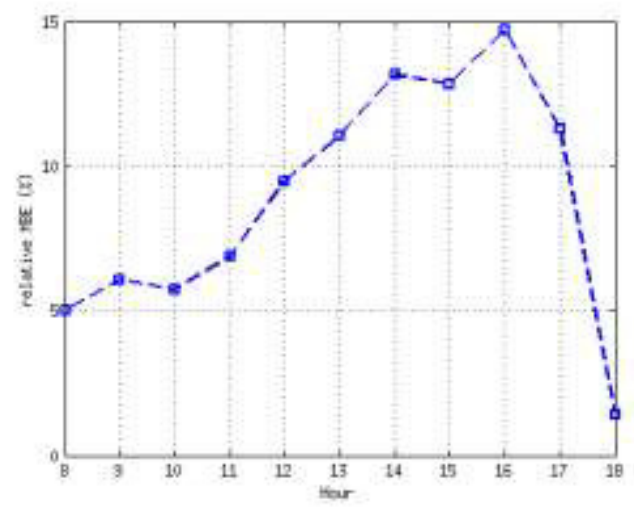

Figure 6: rMBE as a function of time of day

An assumption at Saint Pierre is that there are observations of higher occurence of clear sky in winter than in summer. This is totally verified with the forecast of GHI in 2011 (Figure 7). 


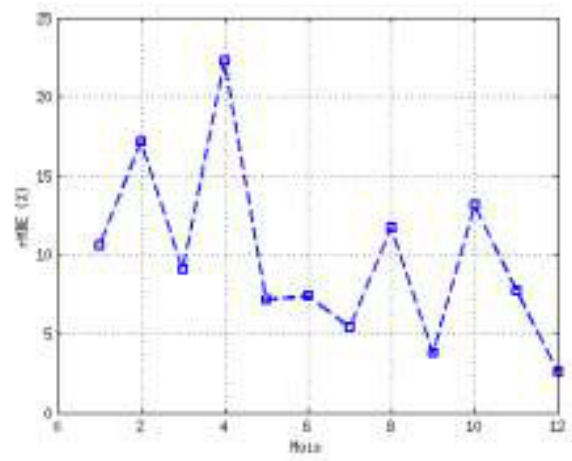

Figure 7: rMBE as a function of month

\subsection{Kalman filtering evaluation}

Results of postprocessing hour by hour forecasted GHI from WRF with our Kalman filtering application is illustrated by Figure 8. It shows that Kalman filtering improves accuracy of forecasted GHI from WRF. In fact, we obtain a rRMSE of $22.33 \%$ and a MBE of . The graphical representation of the MBE depending on the forecasted CSI and the SZA in Figure 5 allowed us to conclude that the Kalman filtering algorithm corrects high underestimation and overestimation.

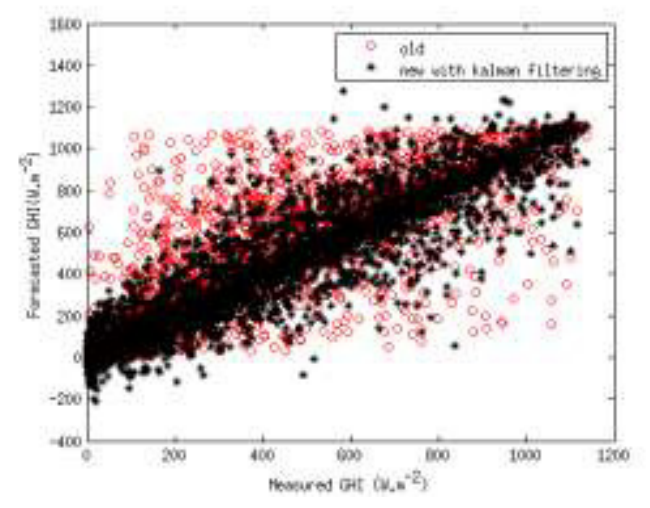

Figure 8: Comparison of WRF accuracy to WRF + Kalman.

Accuracy of WRF model, persistence forecasts and Kalman filtering applied to WRF forecasts are summarized in Table 1. Comparison of GHI forecasted and GHI measured for a partially cloudy day with several methods is illustrated in Figure 10.

Table 1: Accuracy of GHI forecasted

\begin{tabular}{|l|l|l|l|}
\hline & RMSE (rRMSE) & MBE (rMBE) & MAE (rMAE) \\
\hline Persistence & $184.94(34.29 \%)$ & $-13.8(-2.56 \%)$ & $157.76(29.25 \%)$ \\
\hline WRF & $189.82(35.2 \%)$ & $51.44(9.54 \%)$ & $117.68(21.82 \%)$ \\
\hline WRF + Kalman & $120.45(22.33 \%)$ & $1.08(0.2 \%)$ & $79.95(14.82 \%)$ \\
\hline
\end{tabular}




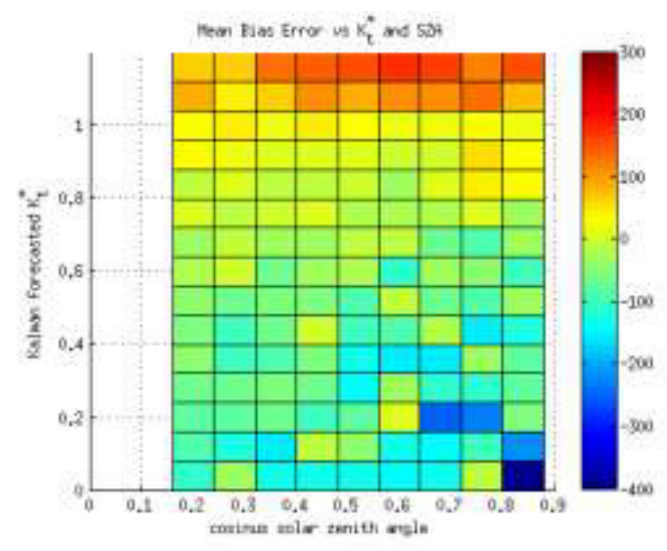

Figure 9: Bias corrected after Kalman filtering

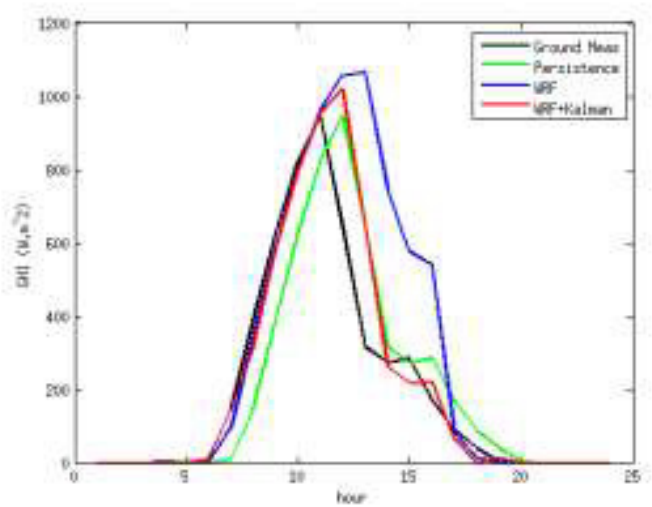

Figure 10: GHI forecasted and measured for one day

\section{Conclusion}

Solar irradiance is the most important weather factor that affects solar energy production. Today, scientists use post-processing methods on the global horizontal irradiance (GHI) forecast from Numerical Weather Prediction (NWP) model to establish reliable planning of energy production.

In this work, hourly GHI forecasts from the WRF model were tested, in terms of the rMBE and rRMSE, against ground measurements collected at ground station at Saint Pierre (La Reunion) along one years from January to December 2011. We compare WRF forecasts to WRF forecasts. Next, we apply the post-processing methods Kalman filtering to WRF forecasts. Our work focused on rRMSE and rMBE reduction of GHI forecasts hour by hour for the day ahead.

Hourly post processing of GHI forecasted with Kalman filtering gave better results than direct WRF output rRMSE decreased from $35.2 \%$ to $22.33 \%$ and rMBE from $9.54 \%$ to . Accuracy evaluation showed 
that rRMSE was reduced by $13 \%$ by Kalman filtering method compared to WRF forecasts without postprocessing.

The main feature of the post-processing methods used here is that they remove the bias in the GHI forecasts without need for a long historical data archive, as they can adapt to weather changes quickly. It should be noted, however that all the post processing methods used in this study are exclusively based on statistical properties of the forecast errors and do not use the physical properties of solar irradiance.

In subsequent work it would be interesting to consider versions of the WRF forecasts that incorporate bias removal methods based on pattern recognition from GHI, temperature and cloud evolution. These approaches would reduce the forecast RMSE further and avoid a systematic tendency to over or under forecast. They were not considered here since information on cloud evolution was not available.

\section{Acknowledgements}

The authors would like to thank the Reuniwatt Company for its support for the present work.

\section{References}

[1] Hadja-Maimouna Diagne, Mathieu David, Philippe Lauret, and John Boland. Solar irradiation forecasting: state-of-the-art and proposition for future developments for small-scale insular grids. In Proceedings of the World Renewable Energy Forum 2012 (WREF 2012), Denver, USA, May 2012.

[2] E.Lorenz and D. Heinemann. 1.13 - prediction of solar irradiance and photovoltaic power. In Editor in Chief: Ali Sayigh, editor, Comprehensive Renewable Energy, pages 239 - 292. Elsevier, Oxford, 2012.

[3] Richard Perez, Sergey Kivalov, James Schlemmer, Karl Hemker Jr., David Renñ̃f Â@, and Thomas E. Hoff. Validation of short and medium term operational solar radiation forecasts in the us. Solar Energy, 84(12):2161 - 2172, 2010

[4] Elke Lorenz, Johannes Hurka, Detlev Heinemann, and Hans Georg Beyer. Irradiance Forecasting for the Power Prediction of Grid-Connected Photovoltaic Systems. IEEE Journal of Selected Topics in Applied Earth Observations and Remote Sensing, 2(1):2-10, March 2009.

[5] E.Lorenz, J. Remund, S.C. MÃ̃1/4ler, W. TraunmÃf̂̂A1/4ller, Steinmaurer, D. G., J.A. Ruiz-Arias, V.L. Fanego, L. Ramirez, M.G. Romeo, C. Kurz, L.M. Pomares, and C.G. Guerrero. Benchmarking of different approaches to forecast solar irradiance. In 24th European Photovoltaic Solar Energy Conference, 2009.

[6] WC Skamarock, JB Klemp, J Dudhia, and al. A description of the advanced research wrf version 3. Technical Note NCAR/TN-475+STR. Boulder, CO: Mesoscale and Microscale Meteorology Division, National Center for Atmospheric Research, 2008.

[7] National Centers for Environmental Prediction (NCEP). http://www.nco.ncep.noaa.gov/pmb/products/gfs/. (web site), 2012.

[8] NCAR Command Language. http://www.ncl.ucar.edu/. (web site), 2012.

[9] Technical report U.S. Geological Survey U.S. Geological Survey. http://www.usgs.gov. (web site), 2012.

[10] Mathieu David, Hadja-Maimouna Diagne, and Philippe Lauret. Outputs and error indicators for solar forecasting models. In Proceedings of the World Renewable Energy Forum 2012 (WREF 2012), Denver, USA, May 2012.

[11] Sophie Pelland, George Galanis, and George Kallos. Solar and photovoltaic forecasting through post-processing of the global environmental multiscale numerical weather prediction model. Progress in Photovoltaics: Research and Applications, pages $\mathrm{n} / \mathrm{a}-\mathrm{n} / \mathrm{a}, 2011$.

[12] Galanis G, Louka P, Katsafados P, Kallos G, and Pytharoulis I. Applications of kalman filters based on non-linear functions to numerical weather predictions. Annals of Geophysics, 24:2451 - 2460, 2006.

[13] Richard E. Bird and Carol Riordan. Simple Solar Spectral Model for Direct and Diffuse Irradiance on Horizontal and Tilted Planes at the Earth's Surface for Cloudless Atmospheres. Journal of Applied Meteorology, 25:87-97, 1986. 